\title{
Formation complémentaire des médecins du travail des centrales nucléaires
}

\author{
H. LETARD et M. CARRÉ (*)
}

(Manuscrit reçu le 18 janvier 1980)

\begin{abstract}
RÉSUMÉ
Les auteurs décrivent succinctement le programme de formation complémentaire qui est donné aux médecins du travail des centrales nucléaires, dans le cadre du Service général de Médecine du Travail d'E.D.F. Cette formation est, bien entendu, indispensable pour faire face aux problèmes spécifiques qui se posent, mais elle ne fait que compléter la formation apportée par les études médicales et le certificat d'études spéciales de médecine du travail et d'hygiène industrielle.
\end{abstract}

\begin{abstract}
A short description is given of the supplementary training course given to nuclear power plant occupational physicians within the frame of the Division of occupational medicine at Électricité de France. Such training is necessary to deal with the specific problems involved. However, it is only a complement to medical studies and the special degree in occupational medicine and industrial hygiene.
\end{abstract}

En France, nul médecin ne peut exercer la médecine du travail sans posséder le certificat d'études spéciales de médecine du travail et d'hygiène industrielle. Dispensé par les Facultés de médecine possédant une chaire de médecine du travail, cet enseignement s'étend sur une période de 2 années et fait du médecin du travail un spécialiste.

Il est évident que la surveillance du personnel travaillant en zone contrôlée dans les centrales nucléaires pose un certain nombre de problèmes spécifiques liés non seulement à la détermination des aptitudes particulières lors de la visite d'embauche et du suivi ultérieur du personnel exposé, mais plus spécialement à la juste appréciation des postes de travail, des niveaux d'irradiation ou de contamination d'ambiance.

Lors d'un incident et, plus encore, à l'occasion d'un accident d'irradiation ou de contamination interne, le médecin du travail doit être à même d'en apprécier rapidement l'importance et de prendre les décisions utiles en tenant

(*) E.D.F., Service général de Médecine du Travail, 22-30, avenue de Wagram, 75008 Paris.

RADIOPROTECTION, VOL. 15 - 0033-8451/1980/67/8 5.00/C Bordas-Dunod. 
compte des difficultés à déterminer exactement l'irradiation reçue ou à estimer, à partir d'une contamination interne, la dose engagée.

Par ailleurs, dans le cadre du rôle général d'information du personnel vis-à-vis des risques ou des nuisances de l'entreprise qui est légalement imparti au médecin du travail, celui-ci doit évidemment bien connaître les notions fondamentales de radiobiologie ou de radiopathologie humaine.

Depuis de longues années déjà, notre souci a été de faciliter l'acquisition des connaissances indispensables au médecin du travail. Dans un premier temps, et alors que les médecins concernés étaient encore très peu nombreux, nous avons dû nous contenter de l'aide et des conseils apportés par l'un d'entre nous à chaque médecin. Dans un second temps, nous avons obtenu du Service de la Production thermique d'Électricité de France que deux demijournées d'activité par semaine soit allouées et payées pendant 18 mois aux médecins des centrales nucléaires pour assurer leur formation. En même temps, nous avons pu réunir les médecins concernés par groupes de 8 à 12 et leur proposer une formation programmée répartie sur 5 à 6 semaines comportant chacune environ 25 heures d'enseignement, celui-ci étant complété par l'équivalent de 2 semaines de stages individuels dans des services médicaux de centrales nucléaires déjà en fonctionnement ou au Centre de Marcoule du C.E.A. $\left.{ }^{1}\right)$.

Le programme de cet enseignement peut, dans ses grandes lignes, être schématisé comme suit.

\section{A. Mathématiques}

Rappel de notions de base portant essentiellement sur la manipulation des puissances de 10, la notion de fonctions (fonction linéaire, fonction de puissance, fonction exponentielle), notion d'intégrale.

\section{B. Physique nucléaire}

Étude de la structure nucléaire, notion d'énergie, radioactivité, période radioactive, rayonnements particulaires $\alpha, \beta$, protons, neutrons, rayonnements électromagnétiques $\gamma$, réactions nucléaires, fission, fusion.

Interaction énergie-matière.

Détection et mesure des rayonnements.

Unités d'activité, unités de dose absolue.

Spectrométrie $\gamma$, anthroporadiamétrie.

\section{Radiobiologie}

Phénomènes d'ionisation.

Réactions radiochimiques fondamentales.

Action directe et indirecte.

(1) Pour cet enseignement il est fait appel à des spécialistes appartenant à E.D.F. ou au C.E.A., ou à une faculté de médecine. 
Radicaux libres, peroxydes.

Effets des rayonnements in vivo : mort cellulaire immédiate ou différée, action sur certaines fonctions cellulaires (ADN-ARN).

Aspects quantitatifs des effets des rayonnements.

Notion de cible et de seuil d'action.

Périodes biologiques-périodes effectives.

Application des rayonnements ionisants en biologie et en médecine au point de vue diagnostic ou thérapeutique (médecine nucléaire).

\section{Radiopathologie}

Effets somatiques : accidents cutanés, oculaires, sanguins, digestifs, action tératogène sur le fotus.

Effets différés : induction d'affections malignes, action génétique.

\section{E. Radioprotection}

Normes de protection, doses maximales admissibles pour les différentes catégories de personnes envisagées.

Concentrations maximales admissibles.

Quantités maximales admissibles.

Problèmes pratiques de mesure d'activité ou de rayonnement.

Écrans de protection.

Perspectives de réglementation future.

Publication CIPR 26 : nouvelle terminologie, nouveaux concepts.

\section{F. Traitements des incidents ou accidents}

a) contamination externe : produits et techniques à mettre en auvre pour la décontamination cutanée.

b) contamination interne : moyens de détermination, anthropogammamétrie, examens radiotoxicologiques des urines, des fèces ou de prélèvement divers (mucus nasal, notamment).

Problèmes pratiques posés par la juste appréciation de la contamination interne et des doses engagées.

Notion de limite de dose délivrée et engagée.

Traitement extemporané ou à long terme de la contamination interne.

Ce programme de formation simplement esquissé dans ses grandes lignes appelle quelques remarques importantes pour bien en mesurer le niveau et la portée. En effet, il ne s'agit pas de faire du médecin du travail un physicien ou un ingénieur. Dans ce domaine, nous avons simplement voulu qu'il soit un interlocuteur valable pour le chef de centrale et les ingénieurs comme pour le personnel, afin d'être à même de comprendre la portée de teile ou telle manæuvre ainsi que les difficultés techniques qu'elle peut comporter.

Au plan de la radiobiologie et de la radioprotection, il importe d'être bien conscient que le calcul des doses après contamination interne n'est jamais

VOL. $15-\mathrm{N}^{\circ} 2$ 
simple; il comporte la mise en auvre de modèles mathématiques plus ou moins exactement adaptés et dans lesquels des paramètres souvent nombreux doivent être pris en considération. Dans ce domaine, il nous est apparu nécessaire que le médecin du travail soit informé des méthodes appliquées, st qu'il soit à même d'en faciliter l'utilisation, mais, dans la majorité des cas, ces calculs sont le fait d'experts, à vrai dire peu nombreux dans chaque pays concerné. C'est à eux que le médecin du travail fera appel en cas de nécessité, c'est avec eux qu'il pourra éventuellement discuter les éléments des modèles à appliquer. Pour autant, le médecin du travail des centrales nucléaires n'est confronté à de tels problèmes qu'à titre exceptionnel. Le même raisonnement s'applique, en cas d'accident grave d'irradiation externe, à l'évaluation des doses reçues et l'on sait que cette évaluation réclame fréquemment des mesures et des calculs effectués par une équipe de travail chargée de reconstituer l'accident et d'établir a posteriori les courbes d'isodoses dont on connaît toute l'importance dans les irradiations sévères. Là encore, le médecin du travail sera un élément de l'équipe, les précisions qu'il pourra apporter seront précieuses, mais en faire un biophysicien strict serait une erreur.

Erreur d'autant plus importante qu'il ne faut pas oublier qu'en plus des problèmes posés par le côté nucléaire de l'installation, l'aspect pratique de surveillance des nuisances conventionnelles telles que le bruit, les solvants, les gaz inertes et bien d'autres éléments de risques sont à prendre en charge par le médecin du travail avec le Comité d'hygiène et de sécurité dont il est de droit un membre important.

On se gardera d'oublier qu'à travers toutes les données techniques qui constituent l'environnement de travail du médecin, celui-ci doit rester un médecin à part entière, conscient de ses responsabilités et de ses techniques médicales propres, et conscient aussi de la disponibilité qu'il se doit de garder vis-à-vis du personnel dans le cadre d'une écoute strictement médicale aux problèmes de pathologie physique ou mentale que n'importe quel agent peut présenter dans n'importe quel domaine.

Autrement dit, il convient, selon nous, de compléter la formation du médecin du travail de centrale nucléaire et non d'en faire un pur spécialiste, éloigné des problèmes médicaux de chaque jour. Ceci explique le fait que, dans un certain nombre de chapitres du programme exposé, est apparu l'expression " notion de " plutôt qu'étude approfondie.

Les connaissances particulières que nous venons d'évoquer ne doivent pas faire sous-estimer la formation de médecin spécialiste qui est celle du médecin du travail. Cette spécialisation, sanctionnée par des examens, demande deux années d'études qui s'ajoutent aux sept années d'études nécessaires à l'obtention préalable du diplôme de Docteur en Médecine.

Toutefois, dans de nombreux domaines industriels, des connaissances spécifiques supplémentaires doivent être acquises par le médecin du travail. Pour n'envisager que le secteur des industries électrique et gazière, des sessions d’informations sur des problèmes particuliers sont périodiquement réalisées, dont les textes publiés sont mis à la disposition de tous nos médecins du travail. 
L'industrie nucléaire réclame, en outre, dans le cadre des installations nucléaires de base, l'apport d'éléments précis de formation complémentaire, soit au plan fondamental (physique, médecine, radiobiologie), soit au plan de la pratique (surveillance du personnel au travail et intervention en cas d'incident ou d'accident corporel de contamination externe ou interne, ou d'irradiation).

En conclusion, on peut considérer qu'un médecin possédant, du fait de ses études, la totalité des capacités médicales aboutissant à l'obtention du Doctorat en Médecine, devient spécialiste en médecine du travail, comme tout autre médecin désireux de se spécialiser dans une branche quelconque, cette spécialisation étant simplement complétée par une formation spécifique qui s'impose dans le cadre de l'exercice de médecin du travail dans les installations nucléaires de base, où, à toutes les autres nuisances observables dans le milieu du travail, s'ajoutent des données particulières concernant les rayonnements ionisants. 


\section{REVUE DES LIVRES}

Advances in radiation protection monitoring, proceedings..., Stockholm, 26-30 June 1978 , Vienne, A.I.E.A., 1979, 790 p. (Prix : 1130 sch. autrichiens).

Ce colloque, dont le thème est repris régulièrement par l'A.I.E.A., a permis de faire le point sur l'état des connaissances en matière de contrôle radiologique à des fins de protection, et a montré les progrès réalisés surtout pour améliorer la qualité, la fiabilitê et le traitement des mesures.

Les communications sont regroupées en quatre grands thèmes. Le premier concerne les méthodes classiques de dosimétrie $\mathrm{X}$ et $\gamma$ du personnel, ainsi que le principe nouveau basé sur la décroissance de la force exercée sur un élément mobile par un électret lorsque celui-ci est irradié. Une large part est également faite à la dosimétrie $\beta$ ou $\beta-\gamma$. Les autres communications portent sur la dosimétrie individuelle des neutrons rapides et montrent le souci accordé à ce problème - lequel reste à résoudre.

Le second thème concerne le contrôle de la contamination - les méthodes spécifiques applicables par exemple au tritium, aux émetteurs $\alpha$ (plutonium, radon...) ou aux mesures in vivo.

Après une session consacrée aux programmes de contrôle des rayonnements, le dernier thème concerne l'étalonnage et pose le problème des rayonnements de référence et des grandeurs que l'on doit utiliser pour étalonner les dosimètres.

L'utilisation d'ordinateurs pour l'exploitation et l'analyse des résultats de dosimétrie, en plein développement, a fait l'objet de cinq communications.

Enfin, un exposé introductif de K. Z. Morgan sur les objectifs du contrôle radiologique et le bilan du colloque présenté par G. CowPER complètent ces actes.

A. M. Beau.

Application of the dose limitation system for radiation protection : practical implications, proceedings of a seminar..., Vienna, 5-9 March 1979, Vienne, A.I.E.A., 1979, 674 p. (Prix : 970 sch. autrichiens).

A l'occasion de la publication des nouvelles recommandations de la C.I.P.R. et de la révision des normes de base de l'A.I.E.A., ce séminaire, organisé conjointement par I'A.I.E.A., I'O.I.T., l'O.M.S., l'Agence pour l'énergie nucléaire de l'O.C.D.E. et la C.I.P.R., a rencontré un très vif succès.

Des communications d'introduction présentent, tout d'abord, les nouvelles recommandations (les principes fondamentaux, le système de limitation des doses, l'évaluation du risque, la justification, l'optimisation). Sont étudiés, ensuite, les nouveaux concepts sous leurs différents aspects ainsi que les conséquences pour la surveillance des travailleurs et les problèmes pratiques de mise en cuvre des nouvelles normes.

Ce séminaire a permis un vaste échange de vues entre porte-paroles des différentes organisations nationales et internationales - C.I.P.R. en particulier - mais aussi responsables de radioprotection, représentants des syndicats de travailleurs, journalistes, etc.

\section{A. M. BeAu.}

Investigations into the emission of carbon-14 compounds from nuclear facilities, Commission des Communautés européennes, doc. $\mathrm{n}^{\circ} \mathrm{V} / 3062 / 78-\mathrm{EN}$. (Ce rapport peut être obtenu sur simple demande à : Commission des Communautés européennes, Direction Santé et Sécurité, V/E/2, bur. A2-78, Bâtiment Jean Monnet, Luxembourg/Kirchberg.)

Ce rapport traite des rejets de carbone 14 par les centrales nucléaires à eau légère et les usines de retraitement - techniques de mesures et d'analyses chimiques, bilan pour la République fédérale d'Allemagne et évaluation des doses à l'échelon local, régional et global.

voL. 15 - No 2 